University of Nebraska - Lincoln

DigitalCommons@University of Nebraska - Lincoln

2011

\title{
Magnetometry and transport data complement polarized neutron reflectometry in magnetic depth profiling
}

\author{
Yi Wang \\ University of Nebraska-Lincoln, ywang1983@hotmail.com \\ Xi He \\ University of Nebraska-Lincoln, lodgehixi@hotmail.com \\ Tathagata Mukherjee \\ University of Nebraska-Lincoln, tatha.muk@gmail.com \\ Mary Lee Fitzsimmons \\ University of Nebraska-Lincoln \\ Sarbeswar Sahoo \\ University of Nebraska-Lincoln, sarbeswar@gmail.com \\ See next page for additional authors
}

Follow this and additional works at: https://digitalcommons.unl.edu/physicsbinek

Part of the Physics Commons

Wang, Yi; He, Xi; Mukherjee, Tathagata; Fitzsimmons, Mary Lee; Sahoo, Sarbeswar; and Binek, Christian, "Magnetometry and transport data complement polarized neutron reflectometry in magnetic depth profiling" (2011). Christian Binek Publications. 76.

https://digitalcommons.unl.edu/physicsbinek/76

This Article is brought to you for free and open access by the Research Papers in Physics and Astronomy at DigitalCommons@University of Nebraska - Lincoln. It has been accepted for inclusion in Christian Binek Publications by an authorized administrator of DigitalCommons@University of Nebraska - Lincoln. 


\section{Authors}

Yi Wang, Xi He, Tathagata Mukherjee, Mary Lee Fitzsimmons, Sarbeswar Sahoo, and Christian Binek 


\title{
Magnetometry and transport data complement polarized neutron reflectometry in magnetic depth profiling
}

\author{
Yi Wang, ${ }^{1}$ Xi He, ${ }^{1}$ T. Mukherjee, ${ }^{1}$ M. R. Fitzsimmons, ${ }^{2}$ S. Sahoo, ${ }^{3}$ and Ch. Binek ${ }^{1, a)}$ \\ ${ }^{1}$ Department of Physics and Astronomy and Nebraska Center for Materials and Nanoscience, \\ Jorgenson Hall, University of Nebraska, Lincoln, Nebraska 68588-0111, USA \\ ${ }^{2}$ Los Alamos National Laboratory, Los Alamos, New Mexico 87545, USA \\ ${ }^{3}$ Seagate Technology, Minneapolis, Minnesota 55435, USA
}

(Received 19 August 2011; accepted 12 October 2011; published online 30 November 2011)

\begin{abstract}
Exchange coupled magnetic hard layer/soft layer thin films show a variety of complex magnetization reversal mechanisms depending on the hierarchy of interaction strengths within and between the films. Magnetization reversal can include uniform rotation, soft layer biasing, as well as exchange spring behavior. We investigate the magnetization reversal of a CoPt/Permalloy/Ta/Permalloy heterostructure. Here, Stoner-Wohlfarth-type uniform magnetization rotation of the virtually free Permalloy layer and exchange spring behavior of the strongly pinned Permalloy layer are found in the same sample. We investigate the complex magnetization reversal by polarized neutron reflectometry, magnetometry, and magneto-transport. The synergy of combining these experimental methods together with theoretical modeling is key to obtain the complete quantitative depth resolved information of the magnetization reversal processes for a multilayer of mesoscopic thickness. (C) 2011 American Institute of Physics. [doi:10.1063/1.3662153]
\end{abstract}

\section{INTRODUCTION}

Depth resolved profiling of field-induced magnetization reversal processes is key for understanding complex reversal mechanisms in exchange coupled magnetic heterolayers. Understanding magnetization reversal in turn is of major importance for the design of functional magnetic heterostructures. ${ }^{1-4}$ Since the advent of magnetic nanostructuring, materials science actively utilizes the relation between structure and magnetic properties.This includes, in particular, emerging interface properties such as interface exchangeinduced unidirectional anisotropy. ${ }^{5,6}$ As a result, modern magnetic materials possess increasing structural complexity enabling significant advances in functionalities, such as an increased energy product in permanent magnets, ${ }^{7}$ improved magnetocaloric properties, ${ }^{8}$ increasing areal storage density in magnetic recording media, and improved sensitivity and scalability of magnetic read heads. ${ }^{9-11}$ The latter in particular are prototypical examples of complex magnetic multilayer structures. Here magnetoresistive properties are tailored through exchange coupling and exchange biasing in magnetic heterostructures.

This manuscript analyzes the magnetization depth profile in a structurally non-trivial magnetic multilayer system. Specifically, we investigate a CoPt biased $\mathrm{NiFe} / \mathrm{Ta} / \mathrm{NiFe}$ heterosystem during field-induced magnetization reversal at room temperature. Our main focus lies on the difference in the magnetization reversal of the Permalloy films. Despite their micro and mesoscopic structural similarity, their magnetic behavior is remarkably dissimilar due to either weak or strong exchange coupling to neighboring films. Special emphasis lies on the reversal behavior in the low field region. Here, the soft Permalloy films reverse virtually

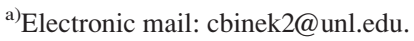

independently and consecutively via qualitative different reversal modes.

We focus on the challenging and hitherto little studied situation where the macroscopic thickness of the overall sample of almost $1 \mu \mathrm{m}$ limits the sensitivity of the otherwise powerful polarized neutron reflectivity (PNR). Although our PNR data provide important qualitative information about the individual reversal mechanisms, quantitative analysis through meaningful fitting of the data is hampered by the challenge of maintaining an intense neutron beam and the competing need for high resolution in measurement of wavevector transfer. ${ }^{12}$ Likewise, straightforward table-top vector magnetometry, such as vector magneto-optical Kerr (MOKE) measurement is not a useful quantitative tool either, because the MOKE information is limited to and averaged over the penetration depth of the light. Therefore, neither depth resolved nor complete integral information of the magnetization vector can be derived from vector MOKE.

Our main objective is to provide evidence that data from integral and scalar magnetometry, when combined with magnetoresistance (MR) data and analyzed in a model motivated by PNR, reveal a quantitative picture of the depth resolved reversal process of the vector magnetization. This approach is of particular interest for the structurally complex CoPt biased $\mathrm{NiFe} / \mathrm{Ta} / \mathrm{NiFe}$ and might as well serve for other structurally complex exchange coupled magnetic heterolayer systems with an intricate depth dependent magnetization reversal.

\section{GENERAL SAMPLE CHARACTERISTICS}

Our $\operatorname{CoPt}(35 \mathrm{~nm}) / \mathrm{Ni}_{0.79} \mathrm{Fe}_{0.21}(450 \mathrm{~nm}) / \mathrm{Ta}(5 \mathrm{~nm}) / \mathrm{Ni}_{0.79}$ $\mathrm{Fe}_{0.21}(450 \mathrm{~nm})$ thin film was grown by room temperature dc magnetron sputtering at a base pressure of $6.7 \times 10^{-8}$ mbar. Deposition took place on a $\mathrm{Si} / \mathrm{SiO}_{2}$ substrate at a pressure of $4 \times 10^{-3}$ mbar. The magnetic multilayer was subsequently 


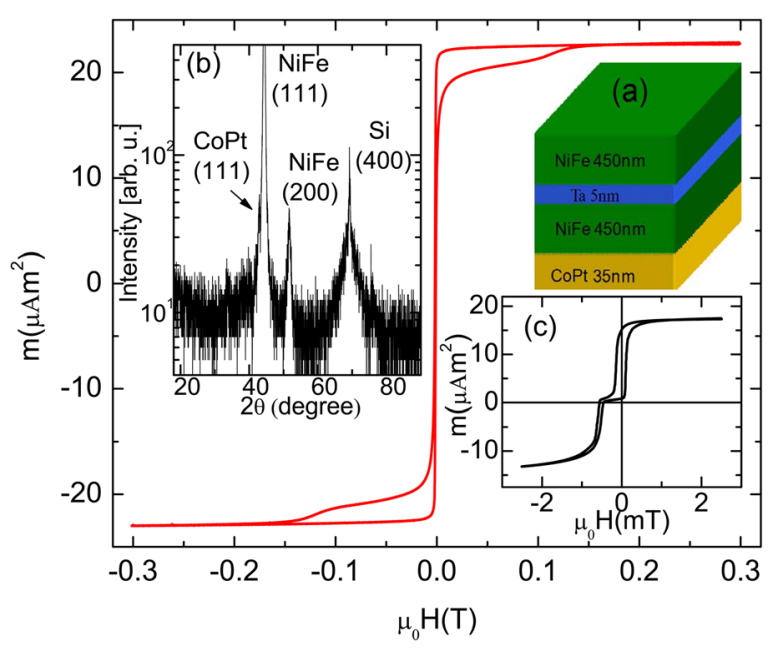

FIG. 1. (Color online) Main panel shows the overall magnetic in-plane hysteresis $m$ vs $\mu_{0} \mathrm{H}$ of the magnetic heterostructure at room temperature. (a) Schematic view of the sample structure. (b) Wide angle X-ray diffraction data showing CoPt (111), NiFe (111), and (200) peaks and the Si (400) substrate peak. (c) Minor magnetic hysteresis loop $m$ vs $\mu_{0} \mathrm{H}$ measured at room temperature after saturation in a field of $0.3 \mathrm{~T}$.

in situ covered with a $10 \mathrm{~nm} \mathrm{Al}_{2} \mathrm{O}_{3}$ protective layer. The sputtering rates, calibrated by X-ray fluorescence (XRF), were 5.5, 1.5 , and $1.5 \AA / \mathrm{s}$ for $\mathrm{NiFe}$, Ta, and CoPt, respectively.

Figure 1(a) shows a cartoon of the magnetic constituents of the layered structure. The inset (b) of Fig. 1 shows the $\theta-2 \theta$ wide angle $\mathrm{X}$-ray diffraction (XRD) data using the $\mathrm{Cu}$ $\mathrm{K}_{\alpha}$ source of our Rigaku D/Max-B diffractometer. Most prominent are the NiFe (111) and (200) peaks together with $\mathrm{Si}$ and sapphire substrate peaks and a CoPt (111) peak, which is the magnetic pinning layer. The Ta interlayer is hardly resolved in the X-ray data since the two orders of magnitude lower thickness result in low scattering intensity.

The mainframe of Fig. 1 displays the overall magnetic hysteresis loop measured for applied magnetic fields $\left|\mu_{0} H\right|<0.3 \mathrm{~T}$, where both Permalloy films and the magnetically harder CoPt film are saturated. The magnetically soft Permalloy films carry the major magnetic moment of the overall heterostructure. In contrast to the magnetically soft Permalloy films where saturation requires only about $\pm 2 \mathrm{mT}$, reversal of the CoPt film sets in at about $\mu_{0} H= \pm 0.15 \mathrm{~T}$. Here the reversible exchange spring behavior discussed below in detail crosses over into irreversibility. ${ }^{1}$ Our investigations focus on the low field region where we measure the successive magnetization reversal of the two Permalloy films henceforth referred to as minor loops. Despite the identical thickness of both Permalloy films, we observe different reversal characteristics.

The Ta spacer layer of $5 \mathrm{~nm}$ thickness gives rise to virtually vanishing magnetic coupling between the Permalloy layers. This behavior is in agreement with the low and rapidly decaying antiferromagnetic coupling reported in Ref. 13 for the $5 \mathrm{~d}$ transition metal Ta. The $\mathrm{Ni}_{0.79} \mathrm{Fe}_{0.21}(450 \mathrm{~nm}) /$ $\mathrm{Ta}(5 \mathrm{~nm}) / \mathrm{Ni}_{0.79} \mathrm{Fe}_{0.21}(450 \mathrm{~nm})$ magnetic heterostructure is reminiscent of trilayers used for magnetoresistive field sensors. However, we show later, that the thickness of the films virtually eliminates the presence of the giant magnetoresistance (GMR) effect $^{14}$ leaving the anisotropic MR (AMR) as the dominating contribution. Due to the absence of sizable magnetic coupling energy across the Ta layer in comparison to the Zeeman energy of the thick Permalloy films, the response of the top Permalloy layer to field is not constrained. The situation for the second Permalloy layer is different. The second layer is strongly coupled to the hard magnetic CoPt layer with in-plane anisotropy. Note that $\mathrm{CoPt}$ is often used for its propensity toward perpendicular magnetic anisotropy, but for our alloy in-plane anisotropy is observed. $^{15}$

\section{INTEGRAL MAGNETIC PROPERTIES}

Angular dependent in-plane magnetic hysteresis loops are measured by means of alternating gradient force magnetometry (AGFM). Those investigations aim at identifying magnetic anisotropies and subsequently allow for detailed low field easy axis hysteresis loop measurements with high field resolution. A major advantage of AGFM over SQUID magnetometry, which we used in addition (not shown) for reference purposes, is the use of normal conducting coils in the AGFM electromagnet. The latter are free from hard to control magnetic flux pinning which is critical when major magnetic reversal features appear in the sub- mT field regime. Therefore, measurements with magnetic field resolution of $\mu_{0} H=0.01 \mathrm{mT}$ are straightforward to perform with AGFM.

Figure 2 shows the results of a systematic study of in-plane anisotropy which allows identifying the easy $\mathrm{NiFe}$ axes. To this end, a sequence of minor loop AGFM measurements has been carried out, where the sample has been rotated in discrete steps relative to the fixed orientation of the applied magnetic field. We show here the qualitative changes occurring for consecutive rotation of $30^{\circ}$. The minor loops shown in Fig. 2(d) are identified as the easy-axis loops of both Permalloy films. This conclusion is based on the following observations. The loop in the quadrants of positive magnetization, henceforth referred to as loop of the top $\mathrm{NiFe}$ layer, shows a gradual steepening in the vicinity of the coercive fields when comparing Figs. 2(a)-2(d). This is consistent with the expected easy axis reversal behavior of a Stoner-Wohlfarth-type free rotating film. ${ }^{16}$ The second NiFe layer shows a very different reversal characteristic in all probed orientations. Below we will strengthen the case that we observe here an exchange spring behavior due to strong exchange coupling with the adjacent CoPt film. In addition to the characteristic gradual shape of the magnetization reversal with absence of coercive fields, the exchange spring behavior is characterized by virtual absence of hysteresis as well. In a very good approximation, this is observed in Fig. 2(d). The far more pronounced hysteretic behavior in Figs. 2(a)-2(c) arises when the applied field is competing with an anisotropy field giving rise to a more complex energy landscape. ${ }^{17}$

\section{POLARIZED NEUTRON REFLECTOMETRY}

PNR measurements were performed using the Asterixreflectometer/diffractometer at the Lujan Neutron Scattering Center (LANSCE). PNR provides a depth profile of both the sample structure and the vector magnetization projected onto 

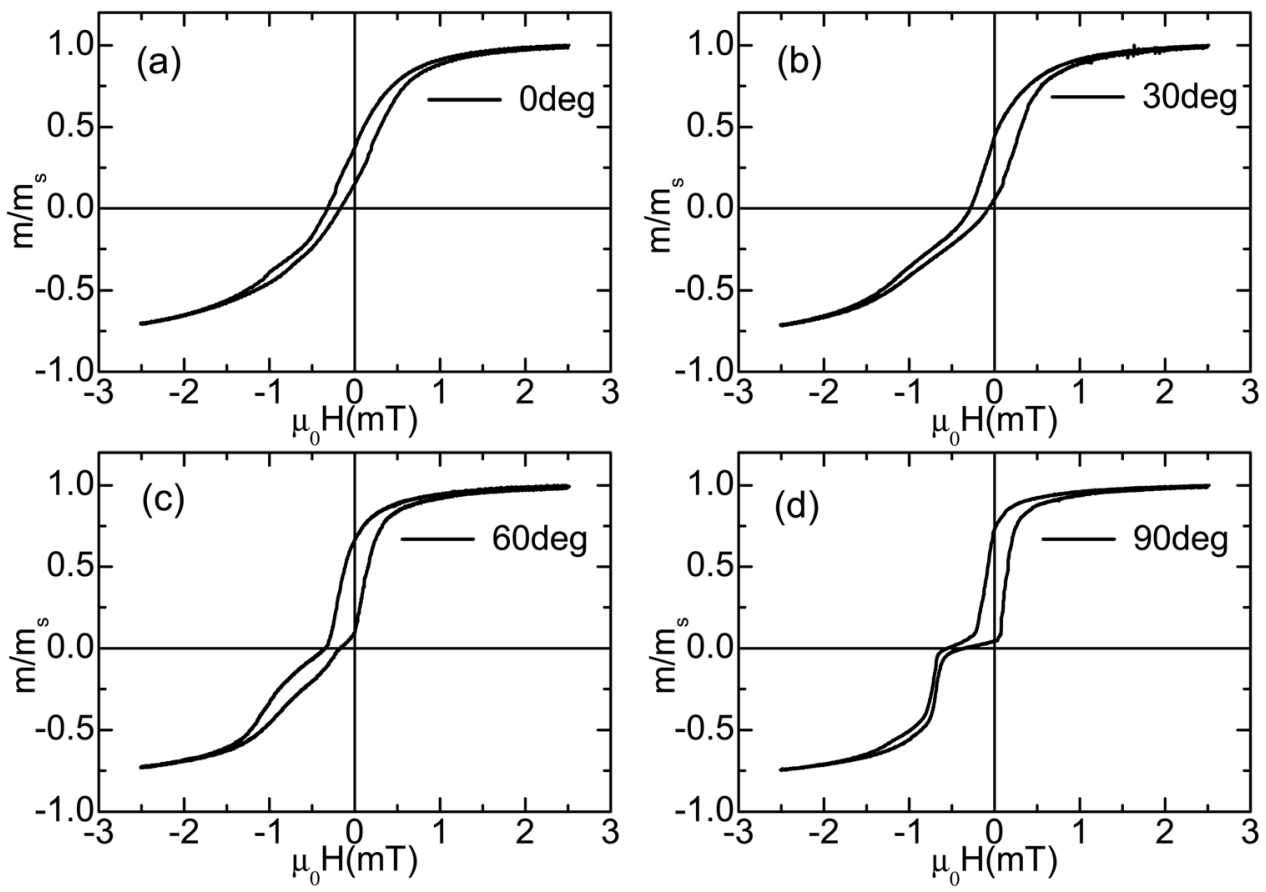

FIG. 2. Normalized minor magnetic hysteresis loops $m / m_{s}$ vs $\mu_{0} H$ for various orientations of the in-plane magnetic field with respect to a fixed reference direction of the sample changing from $0^{\circ}$ (a) to $30^{\circ}$ (b), $60^{\circ}$ (c), and $90^{\circ}$ (d), respectively.

the sample plane. During our experiments, the neutron polarization direction and the applied field were maintained parallel to the uniaxial anisotropy direction in the plane of the sample. We measured all four PNR reflectivities, $R^{++}, R^{--}$, $R^{+-}, R^{-+}$, as a function of the wavevector transfer (difference between the incident and scattered wavevectors) $0<Q_{\perp}<0.1 \AA^{-1}$. The + and - signs designate parallel and antiparallel polarizations of the incident and reflected neutrons relative to the applied magnetic field. Reflectivity $R$ is defined by the ratio of reflected to incident neutron intensity. ${ }^{18}$ Two cross sections contribute to the non-spin-flip (NSF) reflectivity profiles, $R^{++}$(triangles, Fig. 3) and $R^{--}$ (circles, Fig. 3). They originate from the chemical film structure and the component of the magnetization parallel to the applied field. Qualitatively, the difference, $\triangle N S F=R^{++}-R^{--}$ (squares, Fig. 3), is related to the projection of the net sample magnetization averaged over the lateral sample dimensions onto the direction of the applied field, $H_{a},{ }^{19,20}$ i.e.,

$$
\Delta N S F \propto \vec{M} \cdot \vec{H}_{a} / H_{a}=M_{\|} .
$$

The remaining two cross sections create the spin-flip (SF) reflectivities. They are nonzero if the sample changes the neutron beam polarization from spin-up to spin-down $\left(R^{+-}\right)$, and vice versa. For example, the beam polarization will change and SF scattering will be observed, if the magnetic induction vector is perpendicular to the neutron spin, ${ }^{15}$ i.e.,

$$
R^{S F} \propto M_{\perp}^{2} .
$$

Neutron reflectivity profiles, $R$ versus $Q_{\perp}$, were measured for several fields subsequently $(\mathrm{A} \rightarrow 1.40 \mathrm{mT}, \mathrm{B} \rightarrow-0.13 \mathrm{mT}$, $\mathrm{C} \rightarrow-0.22 \mathrm{mT}, \mathrm{D} \rightarrow-0.63 \mathrm{mT}, \mathrm{E} \rightarrow-1.40 \mathrm{mT}$ ) after initial saturation in an in-plane magnetic field of $0.3 \mathrm{~T}$. Data were taken at selected fields correlated with the points A, B, C, D, and $\mathrm{E}$ of the minor loop of $m_{\|}$versus $\mu_{0} H$ (main panel in Fig. 3). Negative applied fields were achieved by reducing the positive applied field to zero, then rotating the sample by $180^{\circ}$ in the zero field, then changing the field by making it more negative. PNR data associated with points A-E of the magnetic hysteresis loop are displayed in individual panels of Fig. 3 and labeled as Figs. 3 A-3 E, respectively.

Point A in Fig. 3 correlates the PNR data taken at $\mu_{0} H_{a}=1.40 \mathrm{mT}$ with the magnetic moment $m_{\|}$measured at the same applied magnetic field in the saturation regime of the minor loop (main panel Fig. 3). The PNR data (Fig. 3A) show a large splitting between the NSF profiles and lack of SF scattering above background. Note near the region where the reflectivity is close to (but not equal to) unity $\mathrm{Q} \sim 0.2 \mathrm{~nm}^{-1}$, $\mathrm{R}^{-}>\mathrm{R}^{++}$. From Eqs. (1) and (2) one concludes saturation of the sample magnetization at $\mu_{0} H_{a}=1.40 \mathrm{mT}$. This is in accordance with the saturation of $m_{\|}$versus $\mu_{0} H$ at point A.

Points $\mathrm{B}$ and $\mathrm{C}$ correspond to $\mu_{0} H_{a}=-0.13 \mathrm{mT}$ and $\mu_{0} H_{a}=-0.22 \mathrm{mT}$ when lowering the field along the descending branch of the hysteresis loop which is related to the magnetization reversal of the top NiFe layer. $\triangle \mathrm{NSF}$ goes virtually to zero when decreasing the field along the path $\mathrm{B} \rightarrow \mathrm{C}$. The SF signal in turn is at maximum at point $\mathrm{B}$ and decreases on approaching point $\mathrm{C}$. The decrease of $\triangle \mathrm{NSF}$ indicates a decrease of net $M_{\|}$. In order to discriminate whether this decrease is related to a decrease in the magnitude of the magnetization vector or a rotation of the latter we refer to the evolving SF signal which implies that the film has magnetization perpendicular to the applied field. With the combination of these two findings, coherent rotation of the NiFe is a plausible mechanism.

Points D and E correspond to $\mu_{0} H_{a}=-0.63 \mathrm{mT}$ and $\mu_{0} H_{a}=1.40 \mathrm{mT}$ when lowering the field further entering 

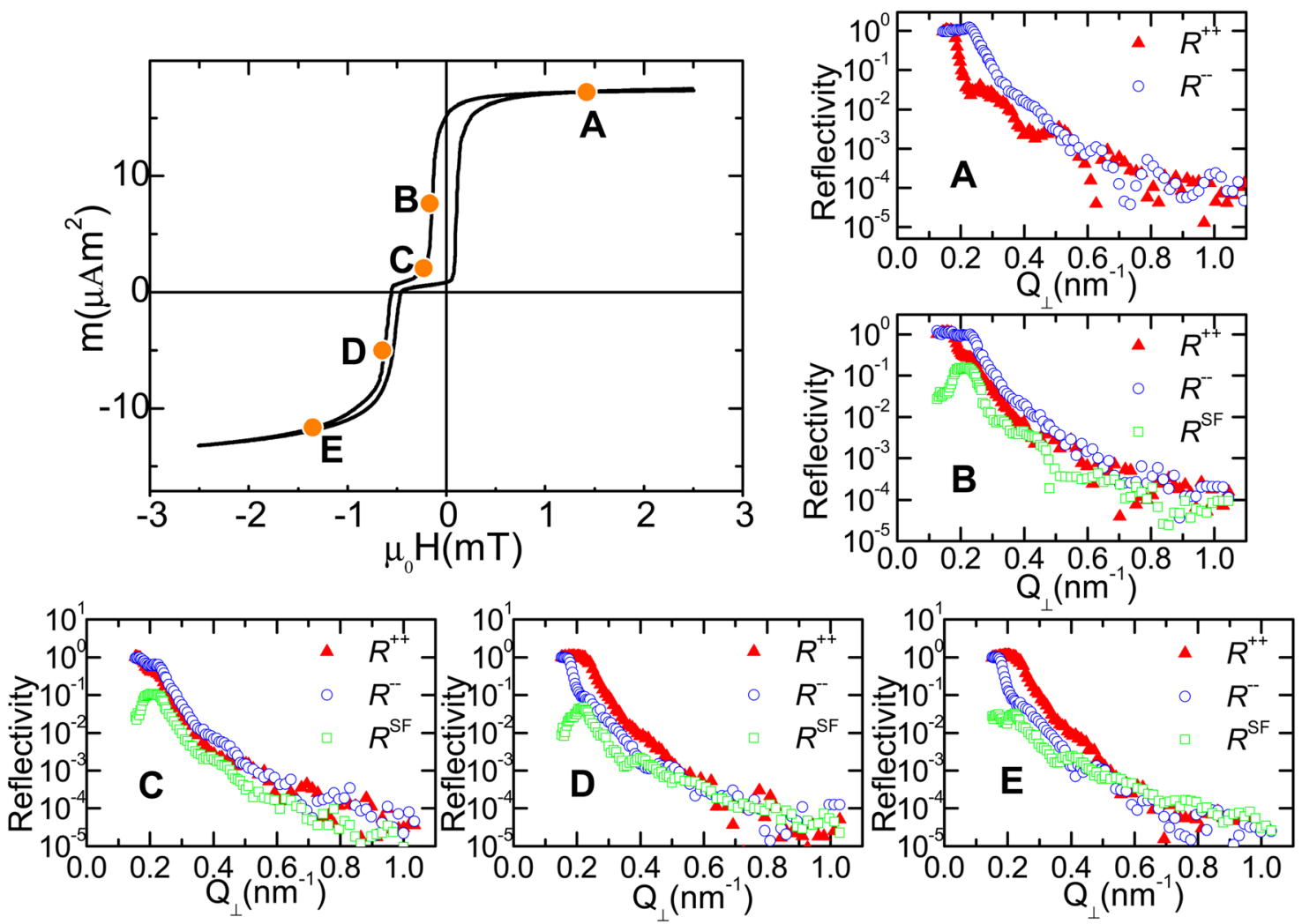

FIG. 3. (Color online) Main panel shows the magnetic minor loop hysteresis $m$ vs $\mu_{0} \mathrm{H}$ with specific highlighted magnetization states A, B, C, D, and E on the descending branch of the loop. Panel A shows the PNR data, $R^{++}$(triangles) and $R^{--}$(circles) vs $Q_{\perp}$, measured at the magnetization state A of the hysteresis loop. Panel B shows $R^{++}$(triangles) and $R^{--}$(circles) vs $Q_{\perp}$ together with the spin flip reflectivity $R^{\mathrm{SF}} \mathrm{vs} Q_{\perp}$ (squares) at the magnetization state $\mathrm{B}$. Panels $\mathrm{C}$, $\mathrm{D}$, and $\mathrm{E}$ show the same set of reflectivities for the corresponding magnetization states $\mathrm{C}, \mathrm{D}$, and $\mathrm{E}$.

the reversal regime of the lower NiFe layer. Remarkably, for both points $\mathrm{D}$ and $\mathrm{E}$ the $R^{++}$signal is larger than the signal of the $R^{--}$channel in contrast to the previous points. The direction of the field in the laboratory frame, to which the polarization of the neutron beam is defined, remained unchanged throughout the experiment, because only the sample was rotated. If the applied field had been sufficient to reverse the magnetizations of the two Permalloy layers and the CoPt layer, then the reflectivities for Point $\mathrm{E}$ would have been identical to those of Point A. In fact, they are not the same, therefore, the magnetization of one or more of the magnetic layers did not fully reverse. The SF intensity on the other hand does not significantly change along the path $\mathrm{D} \rightarrow \mathrm{E}$. This leads to the conclusion that the magnitude of the average component of magnetization perpendicular to the applied field remains virtually constant. This is the fingerprint of an exchange spring reversal mechanism in the intermediate field regime and in contrast to the uniform rotation mode observed for the top layer.

The PNR data set the stage for the understanding of the magnetization reversal. However, the mesoscopic thickness of the films hinders quantitative analysis of the PNR data. ${ }^{21}$ Of particular interest is the depth profile of the lower $\mathrm{NiFe}$ layer which could be quantified by the depth and magnetic field dependent rotation angle of the magnetization. We implement a complementary analysis using integral magnetometry data as input for a quantitative modeling of magnetotransport data. Next we show that this combination provides the quantitative information about the magnetic depth profile of the sample when using the qualitative understanding of the PNR data as the basis for our model.

\section{OUTLINE OF THE LOGICAL STRUCTURE OF THE DATA ANALYSIS}

In the sections that follow, we describe in detail a process to obtain the vector magnetization depth profile. The program of the data analysis outlined below is summarized in an activity chart displayed in Fig. 4. First, we utilize the model assumptions of uniform magnetization rotation of the top $\mathrm{NiFe}$ layer to fit the MR data associated with this rotation. As the input of this MR fit, we use the magnetization $m_{\|}$versus $\mu_{0} H$. In order to simplify the analysis we use an empirical functional form which describes $m_{\|}$versus $\mu_{0} H$ with sufficient accuracy. Evidence for uniform rotation is provided by a successful fit of the MR data using the assumption of uniform rotation and the experimental magnetization data of the top NiFe layer as input. The fit of the free rotation branch of the MR data provides the resistivity parameters of the heterostructure. These numerical values will be used as fixed input for the fit of the MR associated with the exchange spring behavior. Again magnetization is used as input for the MR fit. Our analysis of the exchange spring behavior starts by fitting the $m_{\|}$versus $\mu_{0} H$ data of the descending branch for $-0.1 \mathrm{~T}<\mu_{0} H<-0.005 \mathrm{~T}$ to a $1 / \sqrt{H}$-dependence. ${ }^{1}$ The latter asymptotic field dependence can be derived from the analytic model of the exchange spring in the limit of a rigid pinning layer. ${ }^{17}$ The successful 


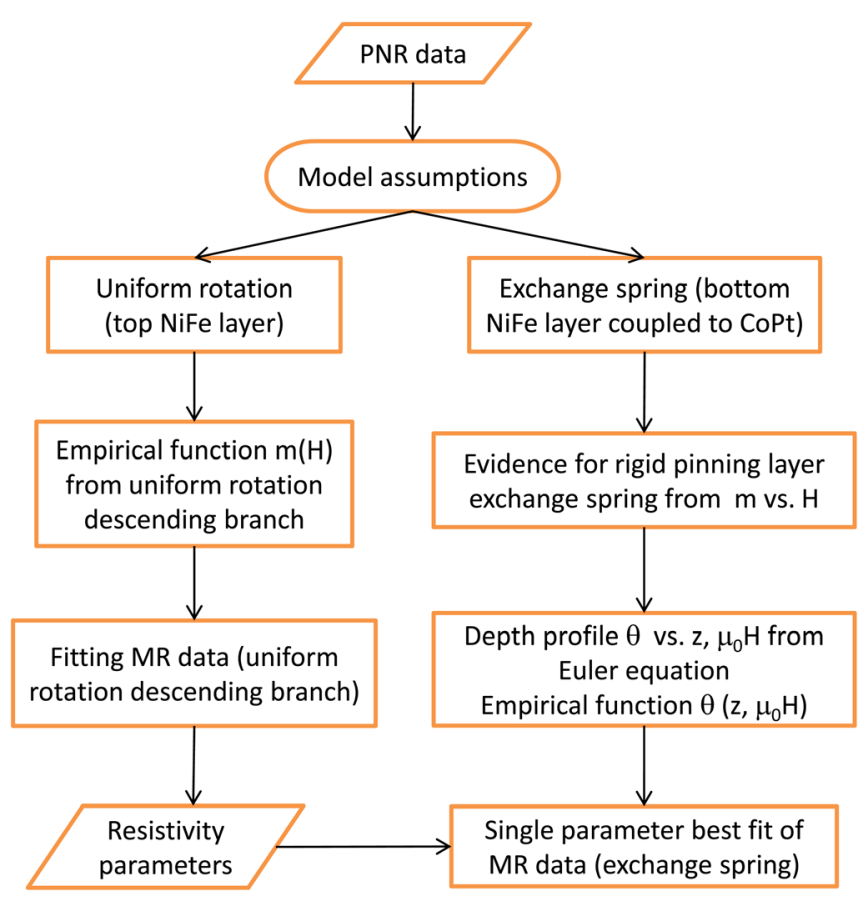

FIG. 4. (Color online) Activity chart visualizing the logical structure of the data analysis which involves PNR, magnetometry, magneto-transport measurements, and development of models for the magnetization reversal mechanisms and their quantitative testing.

fit serves as experimental evidence for the applicability of the rigid pinning approximation. This is in accordance with the fact that the anisotropy of the CoPt pinning layer is much larger than the anisotropy of the soft Permalloy film. Armed with the confirmation that the analytic exchange spring model of Ref. 17 is a good approximation, we now apply this model to calculate the depth dependent magnetization orientation of the exchange spring in the low field part $-2.5 \mathrm{mT}<\mu_{0} H<-0.5 \mathrm{mT}$ of the minor hysteresis loop. The result of this calculation is summarized in the dependence $\theta=\theta\left(z, \mu_{0} H\right)$, where $z$ is the coordinate along the film normal and $\theta$ is the angle between the local magnetization vector and the applied field $\mu_{0} H$. We use this depth dependent magnetization orientation as input for the MR and model the latter leaving only the bottom layer AMR parameter free for a least squares fit, thus ultimately confirming consistency of our model assumptions.

\section{MODELING COHERENT ROTATION AND EXCHANGE SPRING BEHAVIOR}

Figure 5 shows our empirical fit (solid line) of the descending branch of the top NiFe layer magnetization hysteresis (triangles). The functional form $m_{\|}=8.563 \mu \mathrm{Am}^{2}$ $+0.483 \mu \mathrm{Am}^{2} / \mathrm{mT} \mu_{0} H+5.236 \tan ^{-1}\left(3.927+28.972 \mu_{0} H /\right.$ mT) $\mu \mathrm{Am}^{2}$ is a convenient way to import the magnetization data in the subsequent analysis of the MR data discussed in Sec. VII. Note that the physics of uniform rotation is independently supported by the PNR data and ultimately established by the successful fit of the MR data.

Next we focus on the magnetization reversal of the bottom NiFe layer. The PNR data suggest exchange spring behavior. Likewise the virtual absence of hysteresis of

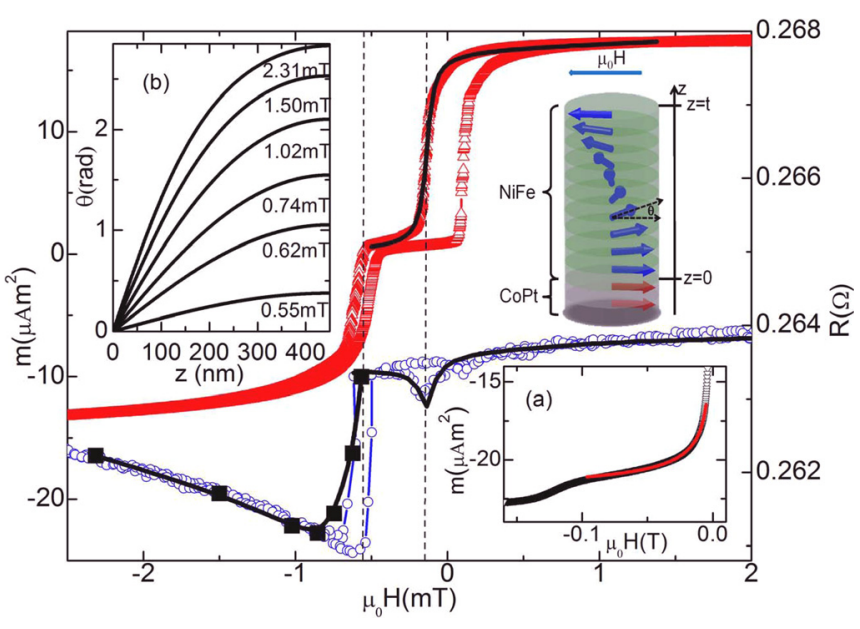

FIG. 5. (Color online) Main panel shows the magnetic minor loop hysteresis $m$ vs $\mu_{0} \mathrm{H}$ (up triangles) with a fit (line) of an empirical function (see text). Open circles show the field dependence of the electric resistance. Solid line with minimum marked by right vertical dashed line is the result of the best fit of the uniform rotation model. Squares are the results of a single parameter fit of the exchange spring model and connected by a spline. The left vertical dashed line indicates the onset of the exchange spring behavior determined by the bending field. Inset (a) shows the descending branch of $m$ vs $\mu_{0} \mathrm{H}$ (down triangles) up to the high field irreversibility region. The line is a best fit of the asymptotic $1 / \sqrt{H}$-dependence predicted by the analytic exchange spring model. Inset (b) shows $\theta$ vs $z$ calculated from numerical solutions of the Euler equation for specific magnetic fields $\mu_{0} H=0.55,0.62$, $0.74,1.02,1.50$, and $2.31 \mathrm{mT}$. The figure in the upper right corner of the main panel is a sketch of the hard layer/soft layer component of the heterostructure. The lower two arrows represent stationary local magnetization of the CoPt pinning layer. The upper 10 arrows indicate the in-plane rotation of local magnetization of NiFe layer. $\theta$ is the angle of the local magnetization (dashed back pointing arrow) with respect to the direction of the pinning magnetization (dashed horizontal arrow). The vertical line is the $z$-axis quantifying the depth coordinate in the NiFe layer starting at $z=0(\mathrm{CoPt} / \mathrm{NiFe}$ interface) and ending at $z=t=450 \mathrm{~nm}$, thickness of the NiFe film.

the lower branch of the $m_{\|}$versus $\mu_{0} H$ data and its gradual approach toward saturation with an onset of irreversibility at high fields strongly support this interpretation. ${ }^{1,9}$ We put the model assumption further to a test by fitting the $m_{\|}$versus $\mu_{0} H$ data of the descending branch for $-0.1 \mathrm{~T}<\mu_{0} H$ $<-0.005 \mathrm{~T}$ to the functional form $m_{\|}=2 m_{s}\left(\sqrt{H_{b} / H}-1\right)$. Here, $m_{s}$ is the saturation moment of the Permalloy film and $H_{b}$ is the bending field strength defined by the onset of magnetization reversal of an exchange spring. We neglect the small contribution of the CoPt pinning layer in order to keep the number of fitting parameters to a minimum. The fit (see inset (a) Fig. 5) provides $m_{s}=11.1 \mu \mathrm{Am}^{2}$ and $\mu_{0} H_{b}$ $=-0.3 \mathrm{mT}$ in reasonable agreement with the direct results from magnetometry. The bending field in particular can be determined with better accuracy from the minor loop data in the main panel of Fig. 5 (left dashed vertical line). The experimental value reads $\mu_{0} H_{b}=-0.54 \mathrm{mT}$. The discrepancy is acceptable when considering the fact that an asymptotic high field expression has been used to extract a parameter which characterizes the onset of the exchange spring behavior in the low-field sub-mT regime. We utilize the experimental value $\mu_{0} H_{b}=-0.54 \mathrm{mT}$ together with the $\mathrm{NiFe}$ saturation moment $m_{s}=11.1 \mu \mathrm{Am}^{2}$ to calculate the exchange constant $A$ of $\mathrm{NiFe}$ from the bending field expression ${ }^{17}$ $\mu_{0} H_{b}=\pi^{2} A L_{x} L_{y} /\left(2 m_{s} t\right)$. Using the sample area $L_{x} L_{y}$ $=5 \mathrm{~mm} \times 10 \mathrm{~mm}=5 \times 10^{-5} \mathrm{~m}^{2}$ and the Permalloy film 
thickness $t=0.45 \mu \mathrm{m}$ we obtain the exchange constant $A=1.1 \times 10^{-11} \mathrm{~J} / \mathrm{m}$ in excellent agree with the literature. ${ }^{9,22}$ Both the success of the fit of the asymptotic field dependence of the magnetization and the determination of the intrinsic Permalloy exchange constant are evidence that the analytic model outlined in Ref. 17 is a valid approximation. Prior to applying it, we verify a necessary condition for all exchange spring models. ${ }^{23}$ The characteristic domain wall width in the reversal layer, here Permalloy, must be larger than the Permalloy film thickness in order to avoid formation of domains. Using the uniaxial anisotropy constant ${ }^{22}$ of $K_{u}=240 \mathrm{~J} / \mathrm{m}^{3}$, the width of a $\pi$-domain wall $\delta=\pi \sqrt{A / K_{u}}$ is estimated to be $0.7 \mu \mathrm{m}$ which is greater than the film thickness $t=0.45 \mu \mathrm{m}$. Thus, formation of domain walls parallel to the sample's surface is not expected.

We use the model of Ref. 17 to calculate the depth profile $\theta=\theta\left(z, \mu_{0} H\right)$. The sketch in Fig. 5 illustrates the geometric parameters $\theta$ and $z$, subsequently used to quantify the depth dependence of the orientation of the local magnetization vector. We outline some details of the calculation yielding $\theta=\theta\left(z, \mu_{0} H\right)$. It emphasizes that the bending field $\mu_{0} H_{b}=-0.54 \mathrm{mT}$ and the boundary conditions are sufficient to constrain $\theta\left(z, \mu_{0} H\right)$. The exchange spring model is based on a magnetic energy density functional which includes exchange and Zeeman energy but neglects anisotropy and magnetostriction. Variation of the energy functional provides the Euler equation. Its solution with the boundary conditions $\theta(z=0)=0$, being the rigid pinning layer approximation, and the free boundary assumption $d \theta /\left.d z\right|_{z=t}=0$, reads $\sin \frac{1}{2} \theta(z)=k \operatorname{sn} u$. Here $u=\frac{\pi z}{2 t} \sqrt{\left|\frac{H}{H_{b}}\right|}$ and $s n u$ is the Jacobi elliptic function. It is defined through $\operatorname{sn} u=\sin \phi$, where $\phi=\phi(u, k)$ is the inverse function of $u(\phi, k)=\int_{0}^{\phi}$ $\frac{d \theta}{\sqrt{1-k^{2} \sin ^{2} \theta}}$. The modulus $k$ entering the elliptic integral reads $k=\sin \frac{1}{2} \theta(t)$. It is physically intuitive that $\theta(z=t)$ and, hence, $k$ are field dependent. The field-dependent $k$ is determined by the solution of $\frac{\pi}{2} \sqrt{\left|\frac{H}{H_{b}}\right|}=\int_{0}^{\pi / 2} \frac{d \theta}{\sqrt{1-k^{2} \sin ^{2} \theta}}$.

Following the above procedure we obtain results $\theta$ versus $z$ from numerical solutions for the specific magnetic fields $\mu_{0} H=0.55,0.62,0.74,1.02,1.50$, and $2.31 \mathrm{mT}$. The $\theta$ versus $z$ data are displayed in the inset (b) of Fig. 5 . The numerical data sets have been fitted with high accuracy to empirical third order polynomials. The latter serve as convenient input functions for the calculation of the MR discussed next.

\section{MAGNETORESISTANCE}

Magneto-transport measurements were carried out at room temperature using four-point probe technique. All MR measurements were performed with the direction of the magnetic field parallel or antiparallel to the electric current.

Figure 5 (circles, main panel) shows the magnetic field dependence of the electric resistance $R$ versus $\mu_{0} H$ in the low field regime $-2.5 \mathrm{mT}<\mu_{0} H<2 \mathrm{mT}$. Here we investigate the correlation between MR and the magnetization reversal as seen in the minor loop hysteresis associated with the NiFe uniform rotation and exchange spring behavior. The prominent features characterizing the magnetization reversal, such as the coercive fields of the uniform rotation and the bending field marking the onset of the exchange spring behavior are found in the resistance data. The correlations are indicated by vertical dashed lines. Remarkably, the exchange spring-type reversal shows a resistance change which is 4 times larger in magnitude than the resistance change created by the uniform rotation of the top NiFe layer. This implies already on a qualitative level that the AMR of the two NiFe layers are significantly different despite the identical layer thicknesses. The overall shape of the $R$ versus $\mu_{0} H$ data for $\mu_{0} H<\mu_{0} H_{b}=-0.54 \mathrm{mT}$ resembles the characteristic features of MR for exchange springs as reported. ${ }^{24,25}$

Next we analyze the MR data quantitatively with particular emphasis on the descending branch of the $R$ versus $\mu_{0} H$ curve. The equivalent circuit ${ }^{26}$ of our heterolayer structure is a parallel combination of the resistances of the top NiFe layer, the Ta layer, the bottom NiFe layer, and the CoPt layer. Within the field regime of interest we consider the $\mathrm{CoPt}$ and the Ta resistances as field-independent. We combine these resistances into an effective parameter $R_{\text {eff. }}$ The $\mathrm{NiFe}$ layers in turn give rise to AMR and, hence, field dependent individual resistances which we label $R_{\text {top }}(H)$ and $R_{\text {bottom }}(H)$ of the top and bottom layer, respectively. With these definitions the total resistance, $R(H)$, of the heterostructure is

$$
R(H)=\frac{R_{\text {top }}(H) R_{\text {bottom }}(H) R_{\text {eff }}}{R_{\text {top }}(H) R_{\text {eff }}+R_{\text {bottom }}(H) R_{\text {eff }}+R_{\text {top }}(H) R_{\text {bottom }}(H)} .
$$

We break the investigation of the MR into two parts which in good approximation can be considered independently from each other due to the lack of NiFe interlayer coupling. In the first part we focus on the uniform rotation and the corresponding field interval $-0.5 \mathrm{mT}<\mu_{0} H<2 \mathrm{mT}$, where the magnetization of the bottom layer remains virtually in its state of positive saturation leaving its resistance value constant $R_{\text {bottom }}\left(-0.5 \mathrm{mT}<\mu_{0} H<2 \mathrm{mT}\right)=R_{0}$. The top NiFe layer, however, gives rise to AMR which, in the case of uniform rotation, can be described as

$$
R_{\text {top }}(H)=R_{0}+\Delta R_{\text {top }} \sin ^{2} \theta,
$$

where in general the anisotropic magnetoresistance, $\Delta R$, is defined as $\Delta R=R_{\|}-R_{\perp}$ with $R_{\|}$and $R_{\perp}$ as the resistance of the NiFe with magnetization oriented parallel $(\theta=0)$ and perpendicular $(\theta=\pi / 2)$ to the electric current, respectively. Since $R_{\perp}$ is typically larger than $R_{\|}$one expects $\Delta R$ to be negative. The angle $\theta$ is directly related to the magnetization, $m(H)$, of the top NiFe layer. Under the assumption of uniform rotation we obtain $\cos \theta=\left(m(H)-m_{s}-m_{s}^{\mathrm{CoPt}}\right) / m_{s}$. We use our analytic empirical function (Fig. 5, solid line, descending branch of $m$ versus $H$ ) to mimic $m(H)$ and fix the CoPt saturation moment as $m_{s}^{\mathrm{CoPt}}=0.64 \mu \mathrm{Am}^{2}$ which can be estimated from the value of the ascending branch of the top $\mathrm{NiFe}$ hysteresis at $H=0$. Substituting the $\theta$-expression into Eq. (4) and substituting the resulting expression into Eq. (3) provides a fitting function which leaves $\Delta R_{\text {top }}, R_{0}, R_{\text {eff }}$, and $m_{s}$ as free fitting parameters. The latter moment can be 
TABLE I. Parameters entering the fit of the MR branch associated with uniform rotation of the top NiFe layer and the exchange spring behavior of the bottom $\mathrm{NiFe}$ layer, respectively. Parameters are indicated as free or fixed.

\begin{tabular}{lccccc}
\hline \hline Parameters & $R_{0}(\Omega)$ & $\Delta R_{\text {top }}(\Omega)$ & $\Delta R_{\text {bottom }}(\Omega)$ & $R_{\text {eff }}(\Omega)$ & $\left.m_{s}(\mu \mathrm{Am})^{2}\right)$ \\
\hline Uniform rotation (UR) & $0.74 \pm 1.3 \%$ & $-0.00341 \pm 3.3 \%$ & Not involved in fit & $0.92 \pm 1 \%$ & $6.97 \pm 1.5 \%$ \\
Exchange spring & Fixed input from UR & Not involved in fit & $-0.034 \pm 3 \%$ & Fixed input from UR & Not involved in fit \\
\hline \hline
\end{tabular}

considered to be the average saturation magnetization of top and bottom NiFe layers which may slightly differ. The result of the least squares fit in the field interval $-0.5 \mathrm{mT}<\mu_{0} H<2 \mathrm{mT}$ is shown in Fig. 5 (line in main panel resembling behavior of MR data, circles) with the characteristic dip in the resistance at the negative coercive field of the top NiFe layer (dashed vertical line). The transport parameters are the crucial result of this fit because they enter the calculation of the MR field-dependence associated with the exchange spring reversal. The fit yields $\Delta R_{\text {top }}=-3.41 \mathrm{~m} \Omega, R_{0}=0.74 \Omega$, and $R_{\text {eff }}=0.92 \Omega$. The negative AMR value $\Delta R / R_{0}=-0.46 \%$ is quite typical for sputtered Permalloy films, ${ }^{27}$ confirming the validity of our approach and thus confirming the Stoner-Wohlfarth-type uniform rotation of the top NiFe layer. Likewise $R_{0}=\rho l /(a t)$ is consistent with the calculation from the resistivity value, ${ }^{28,29}$ $\rho=16 \mu \Omega \mathrm{cm}$, and the sample geometry $l=1 \mathrm{~cm}, a=0.5 \mathrm{~cm}$, and the film thickness $t=0.45 \mu \mathrm{m}$ which yields $R_{0}=0.72 \Omega$ within $3 \%$ accuracy of the fitting result.

Armed with the numerical values of $R_{0}, R_{\text {eff }}$, and $\theta=\theta\left(z, \mu_{0} H\right)$, which quantifies the magnetization orientation in the exchange spring, we now calculate the resistance $R$ versus $\mu_{0} H$ in the field regime $-2.5 \mathrm{mT}<\mu_{0} H<\mu_{0} H_{b}$ $=-0.54 \mathrm{mT}$ leaving only the AMR of the bottom layer as a free fitting parameter. In the field interval of exchange spring behavior the top NiFe layer is saturated and hence, $R_{\text {top }}\left(-2.5 \mathrm{mT}<\mu_{0} H<\mu_{0} H_{b}\right)=R_{0}$ while the bottom $\mathrm{NiFe}$ layer shows field dependent AMR. In order to apply the concept of AMR to the exchange spring we generalize Eq. (4) to the non-uniform situation which reads in the limit $\left|\Delta R_{\text {bottom }} / R_{0}\right| \ll 1, \quad R_{\text {bottom }}(H)=R_{0}+\left(\frac{\Delta R_{\text {botom }}}{t}\right) \int_{0}^{t} \sin ^{2} \theta$ $\left(z, \mu_{0} H\right) d z$. As discussed in Sec. VI, we calculated $\theta\left(z, \mu_{0} H\right)$ for $\mu_{0} H=0.55,0.62,0.74,1.02,1.50$, and $2.31 \mathrm{mT}$ (inset (b) Fig. 5). With this input and the use of Eq. (3) we express the total resistance of our heterostructure at the above field values in terms of the single free parameter $\Delta R_{\text {bottom }}$. A numerical optimization yields $\Delta R_{\text {bottom }}=-0.034 \Omega$ and, hence, $\left|\Delta R_{\text {bottom }} / R_{0}\right| \approx 10 \times\left|\Delta R_{\text {top }} / R_{0}\right|$. The result of the single parameter fit is displayed in Fig. 5 (solid squares). The individual points are connected by an eye-guiding spline. The remarkably increased AMR of the bottom NiFe layer is indeed consistent with findings reported in the literature.

The bottom NiFe layer has been grown on a (111)-textured CoPt seed layer and thus showing pronounced (111)texture as well (see inset (b) Fig. 1). The literature reports an AMR enhancement to above $4 \%$ as a consequence of (111)-textured $\mathrm{NiFe}$ growth with increased grain size ${ }^{30}$ and the strong spin-orbit coupling of $\mathrm{Pt}$ at the interface. ${ }^{31,32}$ When sputtering $\mathrm{NiFe}$ on $\mathrm{Ta}$, referring to the top $\mathrm{NiFe}$ layer, the Pt-induced AMR enhancement is absent, ${ }^{31}$ thus explaining the surprising differences in AMR between our geometrically and chemically similar but micro-structurally different Permalloy films. The quantitative agreement of our single parameter fit with the measured $R$ versus $\mu_{0} H$ data is the final independent confirmation of the model assumptions.

Parameters involved in the MR analysis of the uniform rotation and exchange spring part are summarized in a Table I emphasizing the structure of the analysis. A fit of the StonerWohlfarth-type rotation provides resistance parameters which serve as fixed input in the one parameter fit of the exchange spring behavior.

\section{CONCLUSIONS}

We investigate the exchange coupled thin film multilayer system $\mathrm{CoPt}(35 \mathrm{~nm}) / \mathrm{Ni}_{0.79} \mathrm{Fe}_{0.21}(450 \mathrm{~nm}) / \mathrm{Ta}(5 \mathrm{~nm}) /$ $\mathrm{Ni}_{0.79} \mathrm{Fe}_{0.21}(450 \mathrm{~nm})$ as an example of a complex artificially structured magnetic material. The structural complexity gives rise to an intricate magnetization reversal behavior involving Stoner-Wohlfarth-type uniform rotation and exchange spring behavior. We investigate the magnetization reversal by polarized neutron reflectometry, integral magnetometry, and magneto-transport methodologies. We show that the synergy of combining polarized neutron reflectometry, magnetometry, and magneto-transport provides a complete picture with quantitative depth resolved information of the magnetization reversal. We suggest this approach as a general pathway for quantitative analysis of mesoscopic magnetic heterolayer systems with complex depth dependent magnetization reversal.

\section{ACKNOWLEDGMENTS}

Work at the University of Nebraska-Lincoln is supported by NSF through Career DMR-0547887, MRSEC DMR 0213808 and by the NRC/NRI supplement to MRSEC. This work was supported by the Office of Basic Energy Science, U.S. Department of Energy, BES-DMS funded by the Department of Energy's Office of Basic Energy Science. Los Alamos National Laboratory is operated by Los Alamos National Security LLC under DOE Contract No. DE-AC52-06NA25396.

${ }^{1}$ E. E. Fullerton, J. S. Jiang, M. Grimsditch, C. H. Sowers, and S. D. Bader, Phys. Rev. B 58, 12193 (1998).

${ }^{2}$ Y. Liu, S. G. E. teVelthuis, J. S. Jiang, Y. Choi, S. D. Bader, A. A. Parizzi, H. Ambaye, and V. Lauter, Phys, Rev. B 83, 174418 (2011).

${ }^{3}$ K. V. O'Donaovan, J. A. Borchers, C. F. Majkrzak, O. Hellwig, and E. E. Fullerton, Phys. Rev. Lett. 88, 067201 (2002).

${ }^{4}$ S. Roy, M. R. Fitzsimmons, S. Park, M. Dorn, O. Petracic, I. V. Roshchin, Z. Li, X. Batlle, R. Morales, A. Misra, X. Zhang, K. Chesnel, J. B. Kortright, S. K. Sinha, and I. K. Schuller, Phys. Rev. Lett. 95, 047201 (2005).

${ }^{5}$ Ch. Binek, S. Polisetty, Xi He, and A. Berger, Phys. Rev. Lett. 96, 067201 (2006).

${ }^{6}$ Xi He, Yi Wang, Ning Wu, A. N. Caruso, E. Vescovo, K. D. Belashchenko, P. A. Dowben, and Ch. Binek, Nature Mater. 9, 579 (2010). 
${ }^{7}$ R. Skomski and J. M. D. Coey, Phys. Rev. B 48, 15812 (1993).

${ }^{8}$ T. Mukherjee, S. Sahoo, R. Skomski, D. J. Sellmyer, and Ch. Binek, Phys. Rev. B 79, 144406 (2009).

${ }^{9}$ E. Kneller and R. Hawig, IEEE Trans. Magn. 27, 3588 (1991).

${ }^{10}$ R. Victora and X. Shen, IEEE Trans. Magn. 41, 2828 (2005).

${ }^{11}$ D. Sues, Appl. Phys, Lett. 89, 113105 (2006).

${ }^{12}$ J. F. Ankner and G. P. Felcher, J. Magn. Magn. Mater. 200, 741 (1999).

${ }^{13}$ S. S. P. Parkin, Phys. Rev. Lett. 67, 3598 (1991).

${ }^{14}$ E. Y. Tsymbal and D. G. Pettifor, Solid State Phys. 56, 113 (2001).

${ }^{15}$ M. S. Khatri, H. Schlörb, S. Fähler, and L. Schultz, Phys. Status Solidi A 208, 104 (2011)

${ }^{16}$ R. M. Osgood III, S. D. Bader, B. M. Clemens, R. L. White, and H. Matsuyama, J. Magn. Magn. Mater.182, 297 (1998).

${ }^{17}$ E Goto, N. Hayashi, T. Miyashita, and K. Nakagawa, J. Appl. Phys. 36, 2951 (1965).

${ }^{18}$ C. F. Majkrzak, Physica (Amsterdam) 221B, 342 (1996).

${ }^{19}$ M. R. Fitzsimmons and C. F. Majkrzak, "Application of polarized neutron reflectometry to studies of artificially structured magnetic materials," in Modern Techniques for Characterizing Magnetic Materials, edited by Y. Zhu (Kluwer, Boston, 2005), pp. 107-152.

${ }^{20}$ M. R. Fitzsimmons, P. Yashar, C. Leighton, I. K. Schuller, J. Nogués, C. F. Majkrzak, and J. A. Dura, Phys. Rev. Lett. 84, 3986 (2000).

${ }^{21}$ We attempted a quantitative analysis of the PNR data but encountered two significant problems. First, in order to resolve the fringes from the $1+\mathrm{um}$ thick sample, we required extremely stringent collimation of the neutron beam which given the intensity of the neutron beam would have required far more beam time than was practical. Second, due to the large thickness of the sample, we require at least 128 bit floating point precision in order to calculate the reflectivity in the evanescent region of wavevector transfer. We lack access to such computing resources. Simulation of the PNR data using the depth profile determined from the Stoner-Wohlfarth and exchange spring model assumptions is in qualitative agreement with the PNR data.

${ }^{22}$ F. Suits, IEEE Trans. Magn. 26, 2353 (1990).

${ }^{23}$ H. Kronmüller, and D. Goll, Phys. Status Solidi B, 248, 2361 (2011).

${ }^{24}$ K. Mibu, T. Nagahama, T. Shinjo, and T. Ono, Phys. Rev. B 58, 6442 (1998).

${ }^{25}$ A. Billa, H.B. Braun, J. Magn. Magn. Mater. 272, 1266 (2004).

${ }^{26}$ D. Y. Kim, C. G. Kim, B. S. Park, D. G. Hwang, and S. S. Lee, IEEE Trans. Magn. 35, 2935 (1999).

${ }^{27}$ G. Sung, C. Park, and K. Shin, J. Appl. Phys. 85, 5786 (1999).

${ }^{28}$ A. F. Mayadas, J. F. Janak, and A. Gangulee, J. Appl. Phys. 45, 2780 (1974).

${ }^{29}$ J. Guo, Y. Tzeng, J. van Lierop, S. Chang, and K. Lin, Jpn. J. Appl. Phys. 48, 073003 (2009).

${ }^{30} \mathrm{H}$. Li, J. Ma, G. Yu, S. Long, H. Zhao, and F. Zhu, Chin. Sci. Bull. 48, 1087 (2003)

${ }^{31}$ Y. F. Liu, J. W. Cai, and L. Sun, Appl. Phys. Lett. 96, 092509 (2010).

${ }^{32}$ W. Y. Lee, M. F. Toney, and D. Mauri, IEEE Trans. Magn. 36, 381 (2000). 